Vietnam Journal of Mechanics, VAST, Vol.39, No. 1 (2017), pp. 13-29

DOI:10.15625/0866-7136/7481

\title{
ELASTO-PLASTIC ANALYSIS OF FUNCTIONALLY GRADED METAL-CERAMIC BEAMS UNDER MECHANICAL LOADING
}

\author{
Nguyen Dinh Kien ${ }^{1,2}$, Tran Thi Thom ${ }^{1,2}$, Sergey Alexandrov ${ }^{3}$, Le Thi $\mathbf{H a}^{4}$ \\ ${ }^{1}$ Institute of Mechanics, VAST, 18 Hoang Quoc Viet, Hanoi, Vietnam \\ ${ }^{2}$ Graduate University of Science and Technology, VAST, 18 Hoang Quoc Viet, Hanoi, Vietnam \\ ${ }^{3}$ Institute for Problems in Mechanics, Moscow, Russia \\ ${ }^{4}$ University of Transport and Communications, Hanoi, Vietnam \\ *E-mail: ndkien@imech.vast.vn \\ Received December 02, 2015
}

\begin{abstract}
The elasto-plastic analysis of functionally graded (FG) metal-ceramic beams under mechanical loading by using the finite element method is presented. A bilinear stressstrain relation with isotropic hardening is assumed for elasto-plastic behaviour of metal, and the effective elasto-plastic properties of the functionally graded material are evaluated by using Tamura-Tomota-Ozawa (TTO) model. A nonlinear beam element based on the classical beam theory is formulated and employed in the analysis. The element employed nonlinear von Kámán strain-displacement relationship is derived by using the neutral surface as reference plane. The layer beam approach, in which the plastic rate equation is solved at Gauss points, is adopted in updating the stress and evaluating the element nodal force vector and tangent stiffness matrix. Numerical examples are given to show the accuracy of the derived formulation and to illustrate the effect of the material distribution and plastic deformation on the behavior of the beams. The formation and propagation of plastic zone during the loading process is also examined and highlighted.
\end{abstract}

Keywords: FG beam, elasto-plastic analysis, bilinear model, finite element method.

\section{INTRODUCTION}

Functionally graded materials (FGMs) have received much attention from researchers since they were first initiated by Japanese scientists in 1984. FGMs are produced by varying gradually volume fraction of constituent materials, usually ceramics and metals, in one or more desired spatial directions. The effective properties of the resulted materials exhibit continuous change, and this enables FGMs to overcome the drawbacks such as delamination and stress concentration, which are often met in the multi-layer conventional composite materials. A large number of investigations on the

(C) 2017 Vietnam Academy of Science and Technology 
analysis of functionally graded (FG) structures subjected to different loadings are summarized in a review paper by Birman and Byrd in [1], contributions that are most relevant to the present work are briefly discussed.

Chakraborty et al. [2] derived an exact first-order shear deformable finite beam element for analysis of the thermo-elastic behavior of FG beams. Kadoli et al. [3] proposed a third-order shear deformation finite element formulation for studying the static behavior of FG beams under ambient temperature. Kang and Li [4,5] derived the expressions for the large displacement of nonlinear cantilever FG beams under a transverse tip load or a tip moment by adopting the neutral surface as reference plane. Huang and Li [6] studied the bucking of axially FG Bernoulli columns with varying cross sections by an analytical method. Based on a total Lagrangian formulation, Almeida et al. [7] studied the geometrically nonlinear behavior of FG beams subjected to end forces. Based on the physical neutral surface, Taeprasartsit [8] derived the displacement functions and buckling loads of perfect and imperfect Euler-Bernoulli FG columns. Also using the neutral surface as reference plane, Levyakov [9] derived the elastica solution for FG beam under thermal loading. Based on the third-order shear deformation beam theory, Zhang [10] derived the constitutive equations for studying the nonlinear bending of FG beams. Nguyen [11,12], Nguyen and Gan [13] studied the large deflection of nonuniform FG beams by using the co-rotational finite element formulations.

Elasto-plastic analysis of FG structures has been drawn considerable attention from researchers in recent years. Based on Tamura-Tomota-Ozawa (TTO) model [14] and the finite element code LS-DYNA, Gunes et al. [15] studied the elasto-plastic response of FG circular plates under low-velocity impact loads. Jahromi et al. [16] employed a bilinear tress-strain relationship in modeling the elasto-plastic behavior of an FG rotating disk. The stress field of the disk is then computed with the aid of the finite element package ABAQUS. Huang and Han [17], Huang et al. [18] adopted a multi-linear hardening elasto-plastic model in their study of the elasto-plastic buckling of FG cylindrical shells subjected to axial and torsion loads, respectively. Also using the multi-linear hardening elasto-plastic material model, Zhang et al. [19] studied the buckling behavior of elasto-plastic FG cylindrical shells under a combination of the axial compressive load and external pressure. With the aid of Galerkin method, the authors performed a detail examination on the effects of dimensional parameters and elasto-plastic material properties on the stability region and elasto-plastic interface of the shells. FG beams, which are widely used as structural elements for space vehicles, may also experience plastic deformation due to the exceed loading during the vehicle operation. Analysis of FG beams considering the effect of elasto-plastic deformation, therefore is important topic, and it has been carried out by several authors recently. In this line of work, Nie and Zhong [20] proposed the solutions for stress distribution of curved elasto-plastic FG beams subjected to pure bending. Mahbadi [21] derived the equations for evaluating the collapse loads of FG beams under the axial and/or bending loads. Nguyen et al. [22] studied the postbuckling behavior of FG beams subjected to an eccentric axial load by using the finite element method.

This paper presents a finite element procedure for elasto-plastic analysis of FG beams subjected to various types of mechanical loading. The FGM is assumed to be 
formed from ceramic and metal phases whose volume fraction follows a power-law distribution. A bilinear stress-strain model with isotropic hardening is adopted for the metal, and TTO model is employed to evaluate the effective elasto-plastic properties of the FG beams. A nonlinear beam element based on Euler-Bernoulli beam theory is derived and employed in the analysis. The element adopted the nonlinear von Kámán strain-displacement relation is derived by using the physical neutral surface as reference plane. The layer beam approach, in which the plastic equation is solved at quadrature Gauss points, is employed in updating the axial stress and evaluating the element formulation. The nonlinear equilibrium equations are constructed and solved by an incremental-iterative procedure based on Newton-Raphson method. The effect of material distribution, plastic deformation on the response of the beam is examined and discussed. The formation of propagation of the plastic zone during the loading process, which has not been examined in the above cited references, is also studied and highlighted.

\section{ELASTO-PLASTIC FG BEAMS}

Fig. 1 shows an FG ceramic-metal beam with length $L$, height $h$ and width $b$ in a Cartesian co-ordinate system $(x, z)$. The volume fraction of the ceramic and metal is assume to vary in the thickness direction according to

$$
V_{c}=\left(\frac{z}{h}+\frac{1}{2}\right)^{n}, \quad V_{m}=1-V_{c},
$$

where $z$ is the transverse coordinate, and $n(0 \leq n<\infty)$ is the power-law index. In Eq. (1) and hereafter, the subscripts ' $c$ ' and ' $m$ ' stand for 'ceramic' and 'metal', respectively.

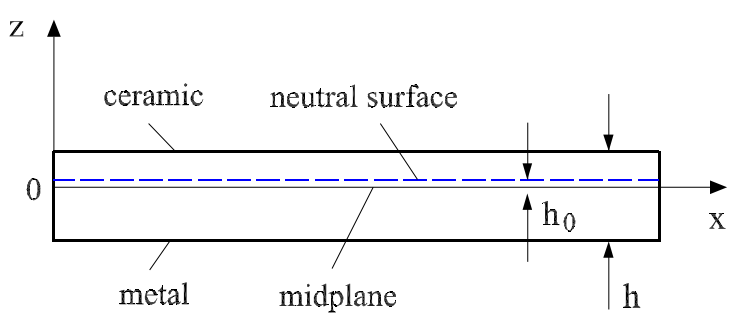

Fig. 1. Geometry of an FG beam

The linear elastic behavior of an FGM is described by Hook's law, and its effective material properties can be evaluated by micromechanics models used in conventional composites. The elasto-plastic behavior of FG ceramic-metal materials is widely described by using TTO model. According to TTO model, the uniaxial stress $(\sigma)$, and uniaxial strain $(\epsilon)$ of a two-phase composite are related to the corresponding average uniaxial stresses and strains of the two constituent materials by [23]

$$
\sigma=\sigma_{c} V_{c}+\sigma_{m} V_{m}, \epsilon=\epsilon_{c} V_{c}+\epsilon_{m} V_{m},
$$


where $\sigma_{c}, \sigma_{m}$ and $\epsilon_{c}, \epsilon_{m}$ denote the average stresses and strains of the ceramic and metal phases. An additional parameter $q$ represented the ratio of stress to strain transfer is introduced as

$$
q=\frac{\sigma_{c}-\sigma_{m}}{\left|\epsilon_{c}-\epsilon_{m}\right|}, 0<q<\infty
$$

The value of $q$ depends on the properties of constituent materials and the microstructural interaction in the composite. Various values of $q$ for FGMs formed from different ceramics and metals are given in Refs. $[15,17]$.

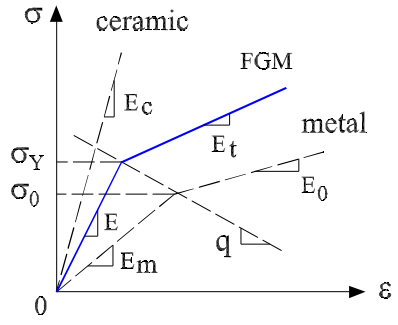

(a)

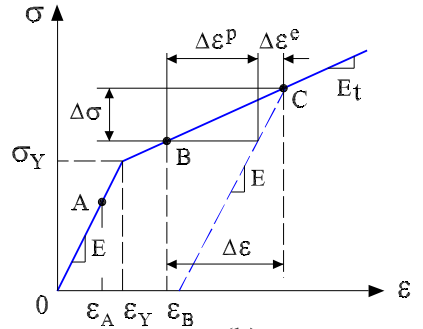

(b)

Fig. 2. A bilinear stress-strain relation for FGM (a), and a stress increment (b)

In TTO model for an FG ceramic-metal material, ceramic phase is assumed to be linearly elastic during its deformation process. Plastic deformation of the composite arose from plastic flow of the metal phase when the stress exceeds its yield limit. Here, a bilinear stress-strain relation with an isotropic hardening is assumed for elasto-plastic behavior of metal. This model, as illustrated in Fig. 2(a), represents a constant tangent modulus $E_{0}$ when the stress in metal phase exceeds its yield limit $\sigma_{0}$. The elasto-plastic behavior of the FG ceramic-metal material also follows a bilinear isotropic hardening model, which represents a tangent modulus $E_{t}$ in the plastic region as depicted in Fig. 2(a) by blue lines. The effective properties such as Young's modulus $(E)$, yield stress $\left(\sigma_{Y}\right)$ and tangent modulus $\left(E_{t}\right)$ of the FGM are evaluated from the material properties of the constituent materials and the parameter $q$ by using TTO model as $[17,23]$

$$
\begin{aligned}
& E=\left[E_{m} V_{m} \frac{q+E_{c}}{q+E_{m}}+E_{c} V_{c}\right] /\left[V_{m} \frac{q+E_{c}}{q+E_{m}}+V_{c}\right], \\
& \sigma_{Y}=\sigma_{0}\left[V_{m}+\frac{q+E_{m}}{q+E_{c}} \frac{E_{c}}{E_{m}} V_{c}\right], \\
& E_{t}=\left[E_{0} V_{m} \frac{q+E_{c}}{q+E_{0}}+E_{c} V_{c}\right] /\left[V_{m} \frac{q+E_{c}}{q+E_{0}}+V_{c}\right] .
\end{aligned}
$$

It is worth to note that at a given value of the power-law index $n$, the effective Young's modulus evaluated by Eq. (4) is slightly smaller than that obtained by Voigt model, namely

$$
E=V_{c} E_{c}+V_{m} E_{m}=\left(E_{c}-E_{m}\right)\left(\frac{z}{h}+\frac{1}{2}\right)^{n}+E_{m} .
$$


The Voigt model is widely employed in nonlinear bending analysis of elastic FG beams $[4,10,12]$. Fig. 3 shows the variation of the Young's modulus and initial yield stress in the beam thickness of an FG beam composed of TiB and Ti. The material properties for $\mathrm{TiB}$ and $\mathrm{Ti}$ are adopted from Ref. [17] as follows: $E_{c}=375 \mathrm{GPa}$ (for TiB), $E_{m}=107 \mathrm{GPa}$, $E_{0}=14 \mathrm{GPa}, \sigma_{0}=450 \mathrm{MPa}$ (for Ti), and $q=4.5 \mathrm{GPa}$.
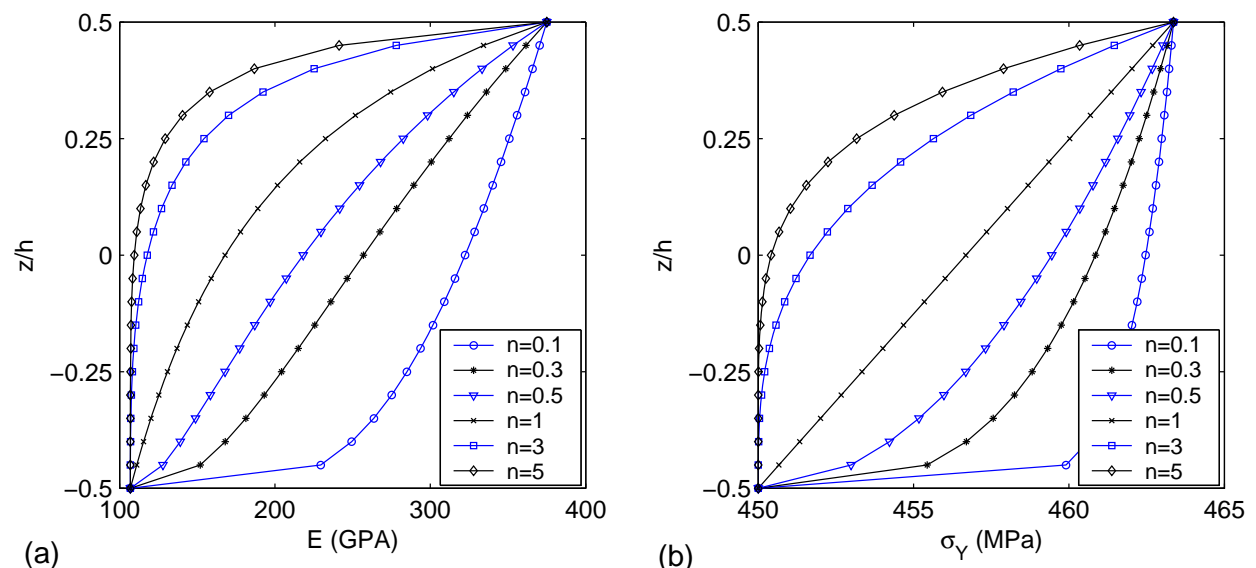

Fig. 3. Variation of Young's modulus (a) and yield stress (b) of FG beam composed of TiB and Ti

The effective Young's modulus defined by Eq. (4) is not symmetrical with respect to the midplane, and the physical neutral surface of the beam is no longer coincident with the midplane. The shift of the physical neutral surface from the midplane, $h_{0}$ (see Fig. 1 ), can be determined from the following equation $[9,10]$

$$
h_{0}=\frac{\int_{-h / 2}^{h / 2} E(z) z d z}{\int_{-h / 2}^{h / 2} E(z) d z}
$$

Integrals in the above equation are hardly computed explicitly for the Young's modulus $E(z)$ defined by Eq. (4), and Simpson's rule is used herewith to determine $h_{0}$.

\section{FINITE ELEMENT FORMULATION}

Adopting the neutral surface as reference plane, the axial and transverse displacements at any point of the beam, $U_{1}(x, z)$ and $U_{3}(x, z)$, based on Euler-Bernoulli beam theory are respectively given by

$$
U_{1}(x, z)=u(x)-\left(z-h_{0}\right) \frac{d w(x)}{d x}, \quad U_{3}(x, z)=w(x),
$$

where $u(x)$ and $w(x)$ are the axial and transverse displacements of a point on the neutral surface, respectively.

The nonlinear von Kámán strain-displacement relationship accounting for moderately large rotation can be adopted for the axial strain in nonlinear bending analysis 
as

$$
\epsilon=\frac{d u}{d x}+\frac{1}{2}\left(\frac{d w}{d x}\right)^{2}-\left(z-h_{0}\right) \frac{d^{2} w}{d x^{2}}=\epsilon_{0}+\left(z-h_{0}\right) \kappa,
$$

where $\epsilon_{0}=d u / d x+\frac{1}{2}(d w / d x)^{2}$ is the membrane strain, and $\kappa=-d^{2} w / d x^{2}$ is the beam curvature.

Assuming the beam is being divided into a number of two-node beam elements with length of $l$. The vector of nodal displacements for a generic element, $(1,2)$, contains six components as

$$
\mathbf{d}=\left\{\begin{array}{ll}
\mathbf{u} & \mathbf{w}
\end{array}\right\}=\left\{\begin{array}{llllll}
u_{1} & u_{2} & w_{1} & \theta_{1} & w_{2} & \theta_{2}
\end{array}\right\}^{T},
$$

where $\mathbf{u}=\left\{\begin{array}{ll}u_{1} & u_{2}\end{array}\right\}^{T}$ and $\mathbf{w}=\left\{\begin{array}{llll}w_{1} & \theta_{1} & w_{2} & \theta_{2}\end{array}\right\}^{T}$ are the vectors of nodal displacements corresponding to the nodal stretching and bending displacements, respectively. It should be noted that the order of the nodal displacements is not necessary as in Eq. (9), but it is convenient to separate the nodal stretching and bending displacements. The virtual work for the beam element reads

$$
\delta V=\int_{V} \sigma \delta \epsilon d V-\int_{0}^{l} q \delta w d x
$$

where $V$ denotes the element volume; $q=q(x)$ is the distributed load acting on the element, and $\sigma$ is the elasto-plastic axial stress as depicted in Fig. 2(a). The bilinear elastoplastic model in the figure can be simply defined by using the instantaneous elasto-plastic modulus $(\tilde{E})$ as $[24,25]$

$$
\tilde{E}=\frac{\partial \sigma}{\partial \epsilon}=\left\{\begin{array}{ll}
E & \text { if } \sigma \leq \sigma_{Y} \\
E_{t} & \text { if } \quad \sigma>\sigma_{Y}
\end{array}\right. \text { or unloading }
$$

The displacements inside the element are interpolated from the nodal displacements as follows

$$
u=\mathbf{N}_{u}^{T} \mathbf{u}, w=\mathbf{N}_{w}^{T} \mathbf{w},
$$

where $\mathbf{N}_{u}^{T}=\left\{\begin{array}{lll}N_{u 1} & N_{u 2}\end{array}\right\}, \mathbf{N}_{w}^{T}=\left\{\begin{array}{llll}N_{w 1} & N_{w 2} & N_{w 3} & N_{w 4}\end{array}\right\}$ are the matrices of interpolation functions for $u$ and $w$, respectively. The following linear and cubic Hermite polynomials are respectively employed to interpolate the axial and transverse displacements

$$
N_{u 1}=\frac{l}{2}(1-\xi), \quad N_{u 2}=\frac{l}{2}(1+\xi),
$$

and

$$
\begin{aligned}
& N_{w 1}=\frac{1}{4}\left(2-3 \xi+\xi^{3}\right), \quad N_{w 2}=\frac{1}{8} l\left(\xi^{2}-1\right)(\xi-1), \\
& N_{w 3}=\frac{1}{4}\left(1+3 \xi-\xi^{3}\right), \quad N_{w 4}=\frac{1}{8} l\left(\xi^{2}-1\right)(\xi+1),
\end{aligned}
$$

with $\xi=2 x / l-1$ is a non-dimensional coordinate, and $0 \leq \xi \leq 1$ for $0 \leq x \leq l$.

The beam element based on the axial strain (8) and the interpolation functions (12)(14) encounters the membrane locking. In order to overcome this problem, the effective 
strain, $\epsilon_{\text {eff., }}$ is introduced to replace the membrane strain in Eq. (8) as [26]

$$
\epsilon_{\text {eff. }}=\frac{1}{l} \int_{0}^{l} \epsilon_{0} d x=\frac{1}{l} \int_{0}^{l}\left[\frac{d u}{d x}+\frac{1}{2}\left(\frac{d w}{d x}\right)^{2}\right] .
$$

Using Eq. (12)-(14), one can write the effective strain in the following form

$$
\epsilon_{\text {eff. }}=\mathbf{b}_{u}^{T} \mathbf{u}+\frac{1}{2 l} \mathbf{w}^{T} \int_{0}^{l} \mathbf{b}_{w} \mathbf{b}_{w}^{T} d x \mathbf{w},
$$

where

$$
\mathbf{b}_{u}=\frac{d \mathbf{N}_{u}}{d x}=\frac{d \mathbf{N}_{u}}{d \xi} \frac{d \xi}{d x}=\frac{1}{l} \begin{cases}-1 & 1\}^{T}\end{cases}
$$

and

$$
\mathbf{b}_{w}=\frac{d \mathbf{N}_{w}}{d x}=\frac{d \mathbf{N}_{w}}{d \xi} \frac{d \xi}{d x}=\frac{1}{4 l}\left\{\begin{array}{c}
6\left(\xi^{2}-1\right) \\
l\left(3 \xi^{2}-2 \xi-1\right) \\
-6\left(\xi^{2}-1\right) \\
l\left(3 \xi^{2}+2 \xi-1\right)
\end{array}\right\}
$$

In performing the integral, Eq. (16) gives an explicit form for the effective strain as follows

$$
\begin{aligned}
\epsilon_{\text {eff. }}= & \frac{1}{l}\left(u_{2}-u_{1}\right)+\frac{1}{30 l^{2}}\left[3 l\left(w_{1}-w_{2}\right)\left(\theta_{1}+\theta_{2}\right)\right. \\
& \left.+18\left(w_{1}-w_{2}\right)^{2}+l^{2}\left(2 \theta_{1}^{2}-\theta_{1} \theta_{2}+2 \theta_{2}^{2}\right)\right] .
\end{aligned}
$$

With the introduction of the effective strain, one can write the axial strain in the form

$$
\epsilon=\mathbf{b}_{u}^{T} \mathbf{u}+\frac{1}{2 l} \mathbf{w}^{T} \int_{0}^{l} \mathbf{b}_{w} \mathbf{b}_{w}^{T} d x \mathbf{w}+\left(z-h_{0}\right) \mathbf{c}_{w}^{T} \mathbf{w},
$$

where

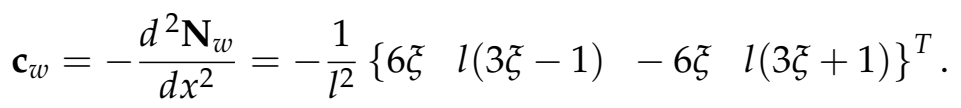

Eq. (20) gives the virtual axial strain in the form

$$
\delta \epsilon=\mathbf{b}_{u}^{T} \delta \mathbf{u}+\left[\mathbf{e}_{w}^{T}+\left(z-h_{0}\right) \mathbf{c}_{w}^{T}\right] \delta \mathbf{w},
$$

where

$$
\mathbf{e}_{w}=\frac{d \epsilon_{\text {eff. }}}{d \mathbf{w}}=\left\{\begin{array}{c}
\frac{1}{10 l}\left[12\left(w_{1}-w_{2}\right)+l\left(\theta_{1}+\theta_{2}\right]\right. \\
\frac{1}{30}\left[3\left(w_{1}-w_{2}\right)+l\left(4 \theta_{1}-\theta_{2}\right)\right] \\
\frac{1}{10 l}\left[12\left(w_{2}-w_{1}\right)+l\left(\theta_{2}+\theta_{1}\right)\right] \\
\frac{1}{30}\left[3\left(w_{2}-w_{1}\right)+l\left(4 \theta_{2}-\theta_{1}\right)\right]
\end{array}\right\} .
$$


The virtual work for the beam element can be now written in the form

$$
\begin{aligned}
\delta V & =\int_{V}\left\{\mathbf{b}_{u}^{T} \delta \mathbf{u}+\left[\mathbf{e}_{w}^{T}+\left(z-h_{0}\right) \mathbf{c}_{w}^{T}\right] \delta \mathbf{w}\right\} \sigma d V-\int_{0}^{l} q(x) \delta w d x \\
& =\mathbf{f}_{\text {in }}^{T} \delta \mathbf{d}-\mathbf{f}_{\mathrm{ex}}^{T} \delta \mathbf{d},
\end{aligned}
$$

where $\mathbf{f}_{\text {in }}$ and $\mathbf{f}_{\mathrm{ex}}$ are respectively the vectors of internal and external nodal forces. By splitting the vector $\mathbf{f}_{\text {in }}$ into stretching and bending parts, $\mathbf{f}_{\text {in }}=\left\{\begin{array}{ll}\mathbf{f}_{u} & \mathbf{f}_{w}\end{array}\right\}$, one can obtain from Eq. (24)

$$
\begin{gathered}
\mathbf{f}_{u}=\left\{\begin{array}{ll}
N_{1} & N_{2}
\end{array}\right\}^{T}=\int_{V} \mathbf{b}_{u} \sigma d V, \\
\mathbf{f}_{w}=\left\{\begin{array}{llll}
Q_{1} & M_{1} & Q_{2} & M_{2}
\end{array}\right\}^{T}=\int_{V}\left[\mathbf{e}_{w}+\left(z-h_{0}\right) \mathbf{c}_{w}\right] \sigma d V .
\end{gathered}
$$

For the distributed load $q(x)$ considered herein, only coefficients corresponding to the nodal bending displacements $\mathbf{w}$ of the external load vector $\mathbf{f}_{\mathrm{ex}}$ are nonzero. Using Eq. (14), a simple expression for the consistent load vector $\mathbf{f}_{\mathrm{ex}}$ defined by Eq. (10) can be easily obtained. Finally, the element tangent stiffness matrix, $\mathbf{k}_{t}$, is obtained by differentiating the element nodal forces with respect to the nodal displacements. To this end, we write $\mathbf{k}_{t}$ in the form

Noting that

$$
\mathbf{k}_{t}=\left[\begin{array}{ll}
\mathbf{k}_{u u} & \mathbf{k}_{u w} \\
\mathbf{k}_{w u} & \mathbf{k}_{w w}
\end{array}\right]=\left[\begin{array}{ll}
\frac{\partial \mathbf{f}_{u}}{\partial \mathbf{u}} & \frac{\partial \mathbf{f}_{u}}{\partial \mathbf{w}} \\
\frac{\partial \mathbf{f}_{w}}{\partial \mathbf{u}} & \frac{\partial \mathbf{f}_{w}}{\partial \mathbf{w}}
\end{array}\right]
$$

$$
\frac{\partial \sigma}{\partial \mathbf{u}}=\frac{\partial \sigma}{\partial \epsilon} \frac{\partial \epsilon}{\partial \mathbf{u}}, \text { and } \frac{\partial \sigma}{\partial \mathbf{w}}=\frac{\partial \sigma}{\partial \epsilon} \frac{\partial \epsilon}{\partial \mathbf{w}} .
$$

From Eqs. (11), (20) and (25) one can obtained the sub-matrices in Eq. (26) in the forms

$$
\begin{aligned}
\mathbf{k}_{u u} & =\int_{V} \mathbf{b}_{u} \mathbf{b}_{u}^{T} \tilde{E} d V, \mathbf{k}_{u w}=\mathbf{k}_{w u}^{T}=\int_{V} \mathbf{b}_{u}\left[\mathbf{e}_{w}^{T}+\left(z-h_{0}\right) \mathbf{c}_{w}^{T}\right] \tilde{E} d V, \\
\mathbf{k}_{w w} & =\int_{V}\left\{\left[\mathbf{e}_{w} \mathbf{e}_{w}^{T}+2\left(z-h_{0}\right) \mathbf{e}_{w} \mathbf{c}_{w}^{T}+\left(z-h_{0}\right)^{2} \mathbf{c}_{w} \mathbf{c}_{w}^{T}\right] \tilde{E}+\mathbf{B} \sigma\right\} d V,
\end{aligned}
$$

in which $\mathbf{B}$ is a symmetric matrix with the following components

$$
\mathbf{B}=\frac{\partial \mathbf{e}^{T}}{\partial \mathbf{d}}=\frac{1}{30 l}\left[\begin{array}{cccc}
36 & 3 l & -36 & 3 l \\
3 l & 4 l^{2} & -3 l & -l^{2} \\
-36 & -3 l & 36 & -3 l \\
3 l & -l^{2} & -3 l & 4 l^{2}
\end{array}\right]
$$

It is easy to verify that for a homogeneous beam, the element nodal force vector and tangent stiffness matrix given by Eqs. (25) and (28) deduce to the formulations previously derived by the first author and his co-workers in [27].

The nonlinear equilibrium equations for the beam constructed from the derived element internal force vector and tangent stiffness matrix can be written in the form [26]

$$
\mathbf{g}(\mathbf{D}, \boldsymbol{\lambda})=\mathbf{F}_{\text {in }}(\mathbf{D})-\mathbf{F}_{\mathrm{ex}}=\mathbf{F}_{\text {in }}(\mathbf{D})-\lambda \mathbf{f}_{\mathrm{ef}}=\mathbf{0} .
$$


In Eq. (30), $\mathbf{g}$ is the out of balance force vector; $\mathbf{D}, \mathbf{F}_{\text {in }}$ and $\mathbf{F}_{\text {ex }}$ are respectively the structural vectors of nodal displacements, internal and external nodal forces; $\mathbf{f}_{\text {ef }}$ is the fixed external loading vector, and $\lambda$ is a load level parameter.

Eq. (30) can be solved by an incremental-iterative procedure based on NewtonRaphson method [26]. Gauss quadrature is employed to compute the force vector and stiffness matrix defined by Eqs. (25) and (28). A simple algorithm for updating stress of one-dimensional elasto-plastic problems described in $[28,29]$ is adopted herein.

\section{NUMERICAL EXAMPLES}

Numerical examples are given in this Section to show the accuracy of the formulated formulation and to illustrate the effect of material distribution and plastic deformation on the behavior of FG beam under various types of loading. Otherwise stated, nine Gauss points along the element length and fifteen Gauss points through the beam thickness are employed in all the computations reported below. The large numbers of Gauss points are used in the beam thickness in order to predict the plastic deformation as soon as it appears near the beam surfaces.

\subsection{Formulation verification}

The present formulation reduces to the one previously derived by Nguyen et al. in [22] for the case of FG beams under an eccentric axial force. In addition, to the authors' best knowledge, there is no investigation the elasto-plastic response of FG beams under distributed loads available in the literature, the verification is focused on the nonlinear bending of elastic FG beams under a distributed load. To this end, the nonlinear bending behavior of a clamped elastic $\mathrm{Si}_{3} \mathrm{~N}_{4}$-SUS304 beam under uniform distributed load $q_{0}$, previously studied by Zhang [10] by using Ritz method, is investigated. In order to analyze the elastic beam by using the present computer code, the yield stress $\sigma_{0}$ is set to a large value in the input data, and thus yielding will not occur. In Fig. 4 the normalized

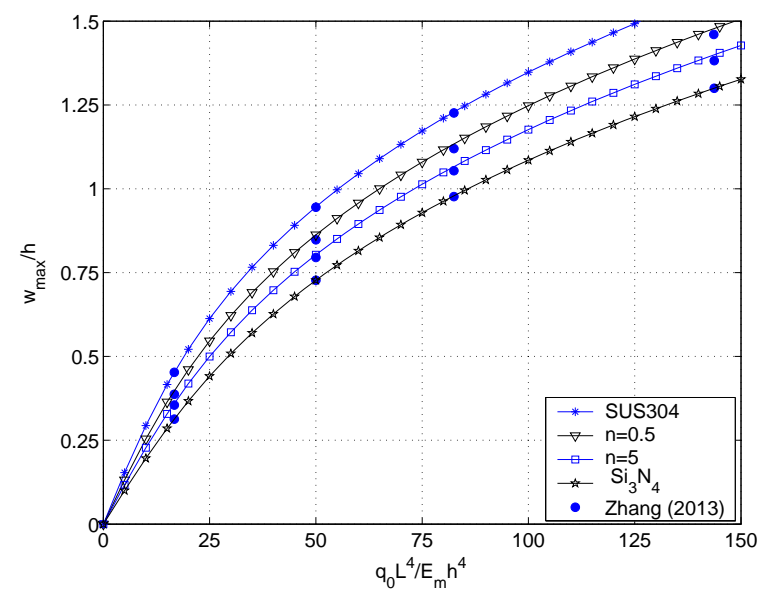

Fig. 4. Normalized applied load versus the dimensionless maximum transverse displacement of clamped elastic $\mathrm{Si}_{3} \mathrm{~N}_{4} /$ SUS304 beam under uniform distributed load 
applied load versus the maximum dimensionless transverse displacement of the beam is depicted for various values of the power-law index $n$, where the result by Zhang is illustrated by small blue circles. A good agreement between the numerical of the present paper with that of Zhang is noted. It is worth to mention that the numerical result in Fig. 4 has been converged by using sixteen elements, and this number of elements is used in all the computations reported below.

\subsection{Clamped beam under distributed load}

An FG beam clamped at both ends (referred to as CC beam) under a uniformly distributed load $q_{0}$ is considered. The beam with square section, $b=h=1 \mathrm{~m}$, and an aspect ratio $L / h=20$ is formed from $\mathrm{TiB}$ and $\mathrm{Ti}$ with the elasto-plastic properties stated in Section 2. In Fig. 5, the relation between the normalized applied load and the normalized maximum deflection of the $\mathrm{CC}$ beam obtained by both the elastic and elasto-plastic analyses is depicted for two indexes, $n=0.5$ and $n=5$. The effect of plastic deformation on the nonlinear bending is clearly seen from the figure. The transverse displacement obtained by the elasto-plastic analysis is considerably larger than that obtained by the elastic analysis, and this is more pronounced at the higher load amplitude. The effect of the material distribution on the nonlinear bending of the elasto-plastic beam is similar to that of the elastic beam, in which the maximum displacement is larger for the beam associated with a higher index $n$.

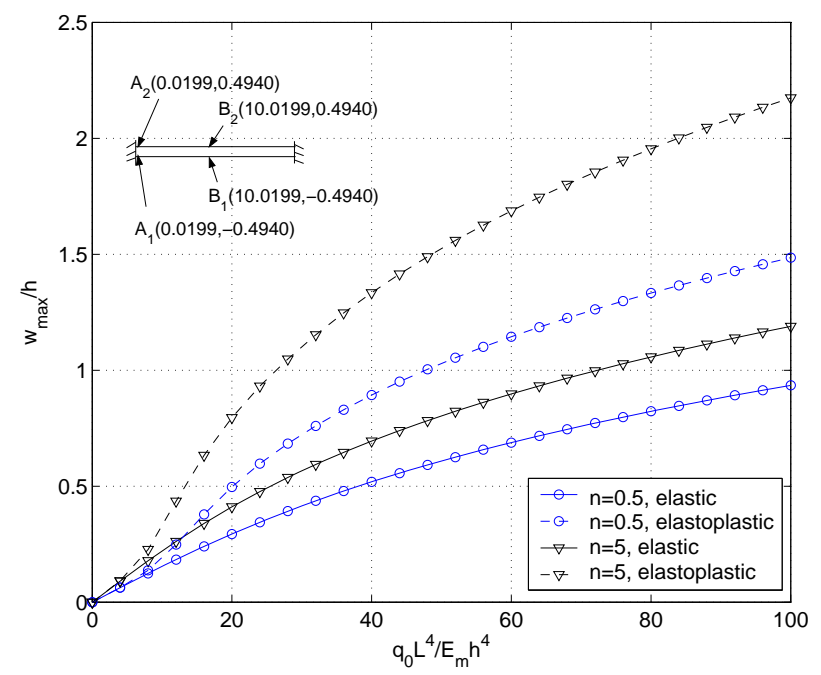

Fig. 5. Relation between normalized load and dimensionless maximum deflection of CC beam

In order to study how the yielding occurs and the plastic deformation propagates, the axial stresses at lower and upper Gauss points of the clamped and middle section of the CC beam, namely points $A_{1}, A_{2}, B_{1}$ and $B_{2}$ in Fig. 5 , are monitored during the loading process. The co-ordinates of the sampling Gauss points are shown in the upper left corner of Fig. 5. Tab. 1 lists the stress at the sampling points at various values of the load amplitude. The results given in the table were obtained with a load increment 
Table 1. Axial stress $\left(\times 10^{8} \mathrm{~N} / \mathrm{m}^{2}\right)$ at Gauss points $A_{1}, A_{2}, B_{1}, B_{2}$ in Fig. 5 of $C C$ beam

\begin{tabular}{|c|c|c|c|c|c|c|c|}
\hline \multicolumn{8}{|c|}{$q_{0}(\mathrm{kN} / \mathrm{m})$} \\
\hline$n$ & Point & 1000 & 1500 & 2000 & 2500 & 3000 & 3500 \\
\hline \multirow[t]{4}{*}{0.2} & $A_{1}$ & 1.2127 & 1.8211 & 2.4305 & 3.0457 & 3.6752 & 4.3372 \\
\hline & $\mathrm{A}_{2}$ & -2.4331 & -3.6427 & -4.8428 & -6.0299 & -7.2494 & -8.5388 \\
\hline & $\mathrm{B}_{1}$ & -0.6116 & -0.9146 & -1.2156 & -1.5146 & -1.8117 & -2.1077 \\
\hline & $\mathrm{B}_{2}$ & 1.2492 & 1.8792 & 2.5124 & 3.1491 & 3.7912 & 4.4409 \\
\hline \multirow[t]{4}{*}{0.5} & $\mathrm{~A}_{1}$ & 1.2335 & 1.8524 & 2.4748 & 3.1183 & 3.7805 & 4.4626 \\
\hline & $\mathrm{A}_{2}$ & -2.7909 & -4.1754 & -5.5134 & -6.8814 & -8.3103 & -9.8110 \\
\hline & $\mathrm{B}_{1}$ & -0.6212 & -0.9283 & -1.2330 & -1.5354 & -1.8357 & -2.1343 \\
\hline & $\mathrm{B}_{2}$ & 1.4373 & 2.1640 & 2.8956 & 3.6355 & 4.3863 & 5.1267 \\
\hline \multirow[t]{4}{*}{2} & $\mathrm{~A}_{1}$ & 1.5332 & 2.3041 & 3.1062 & 3.9517 & 4.5428 & 4.6845 \\
\hline & $\mathrm{A}_{2}$ & -3.4447 & -5.0647 & -6.5526 & -8.1675 & -9.8875 & -12.0164 \\
\hline & $\mathrm{B}_{1}$ & -0.7701 & -1.1496 & -1.5256 & -1.8988 & -2.2708 & -2.6612 \\
\hline & $\mathrm{B}_{2}$ & 1.7815 & 2.6852 & 3.6025 & 4.5420 & 5.3689 & 6.2808 \\
\hline \multirow[t]{4}{*}{5} & $\mathrm{~A}_{1}$ & 1.6428 & 2.4782 & 3.3678 & 4.2907 & 4.6085 & 4.7955 \\
\hline & $\mathrm{A}_{2}$ & -3.9650 & -5.5256 & -6.9885 & -8.5542 & -10.3605 & -12.7688 \\
\hline & $\mathrm{B}_{1}$ & -0.8234 & -1.2283 & -1.6297 & -2.0313 & -2.4392 & -2.8743 \\
\hline & $\mathrm{B}_{2}$ & 2.0523 & 3.0956 & 4.1626 & 5.0680 & 5.8752 & 6.8106 \\
\hline
\end{tabular}

of $500 \mathrm{kN} / \mathrm{m}$. In the Table, the numbers in bold face are the values of the stress at the Gauss points where yielding has occurred. As seen from the table, yielding at the top and bottom layers (containing the Gauss points) of the FG beam does not occur at the same time during the loading as it happens in an elasto-plastic homogeneous beam. At a given value of the index $n$, yielding, according to TTO model, commences in the layer close to the ceramic surface much earlier that it does in the layer near the metal surface. The formation of the first yielding in the layer close to the ceramic surface found herein is similar to that obtained by Nie and Zhong in Ref. [20], where a curved elasto-plastic FG beam under pure bending was studied. Tab. 1 also shows that the power-law index $n$ influences the first yielding in the FG beam, and the first yielding of the beam associated with a higher index $n$ commences at a lower load amplitude.

In Fig. 6, the plastic zones in some selected elements, namely elements 1, 2, 8, 9,15 and 16, of the CC beam are depicted for an index $n=2$ and various values of the load amplitude $q_{0}$. The figure shows a steady expansion of plastic zones from the outer surfaces into the interior of the beam as the load increases. The plastic propagation from the bottom surface to the interior is, however very different from that from the top surface. Although yielding commences in the layer near the top (ceramic) surface first, the plastic propagation from this surface to interior of the beam, as seen from Fig. 6(c), is 
(a)

\begin{tabular}{|c|c|}
\hline (1) & 2 \\
\hline $\mid$ & : : : : : • \\
\hline |: & \\
\hline
\end{tabular}

element number

(b)
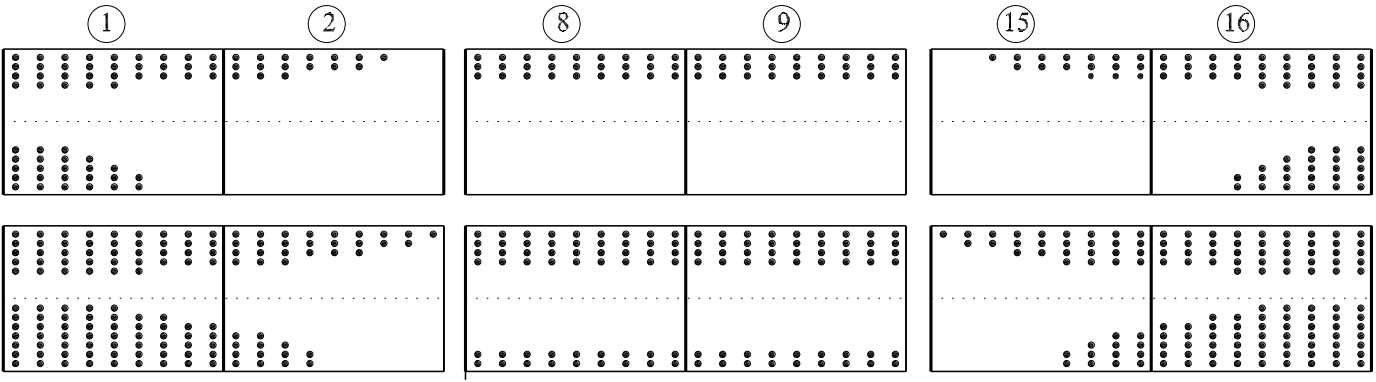

(c)
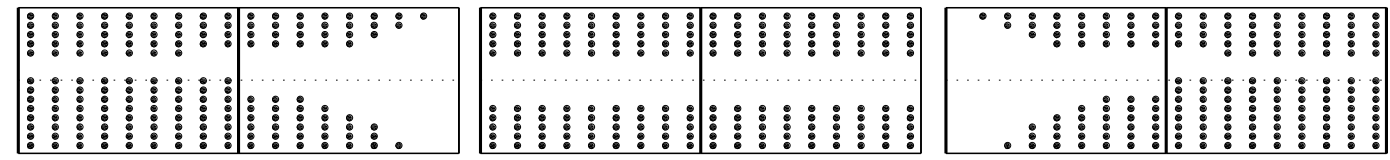

- Gauss points where yielding occurs

Fig. 6. Plastic zone in selected elements of CC beam: (a) $q_{0}=4000 \mathrm{kN}$, (b) $q_{0}=6000 \mathrm{kN} ;$ (c) $q_{0}=8000 \mathrm{kN}(n=2)$

slower than that from the bottom (metal) surface. The propagation of the plastic zone can also be seen from Fig. 7, where the distribution of the axial stress in the beam thickness of clamped section is shown for various value of the load level and for $n=5$. The height of the beam thickness corresponding to the elastic stress gradually decreases as the load amplitude increases. For a give load amplitude, the height corresponding to the elastic stress of the clamped section is smaller than that of the middle section, and this observation is in accordance with the plastic zones depicted in Fig. 6. It should be noted that in order to ensure the smoothness of the curves, thirty one Gauss points in the beam

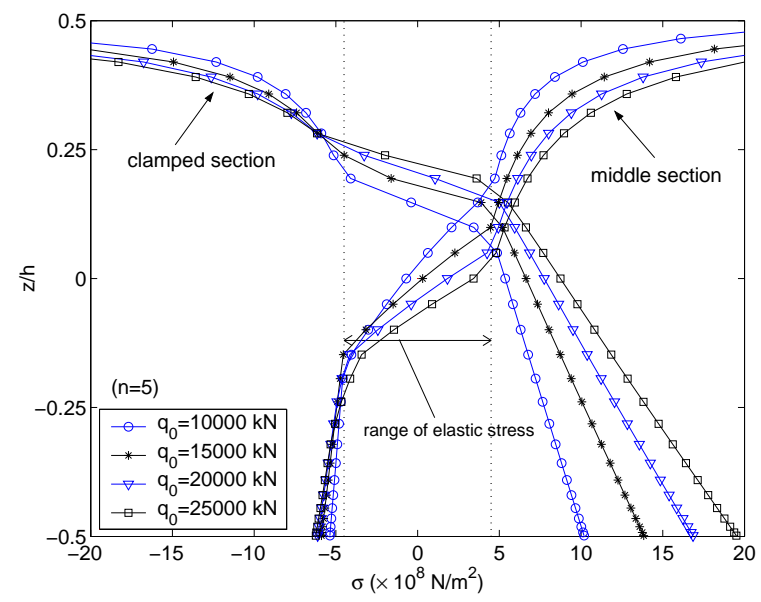

Fig. 7. Thickness distribution of axial stress of clamped section of CC beam $(n=5)$ 
thickness have been used in computing the stress in Fig. 7, and only a part of the obtained stresses is displayed in the figure.

\subsection{Immovable simply supported beam under distributed load}

An immovable simply supported beam (referred to as SS beam) composed of TiB and Ti under a uniform distributed load $q_{0}$ is analyzed this Sub-section. In Fig. 8 the relation between the normalized load and the dimensionless deflection of the SS beam is depicted for various values of the index $n$. As in case of the CC beam, for a given value of the applied load the maximum deflection of the SS beam increases by increasing the

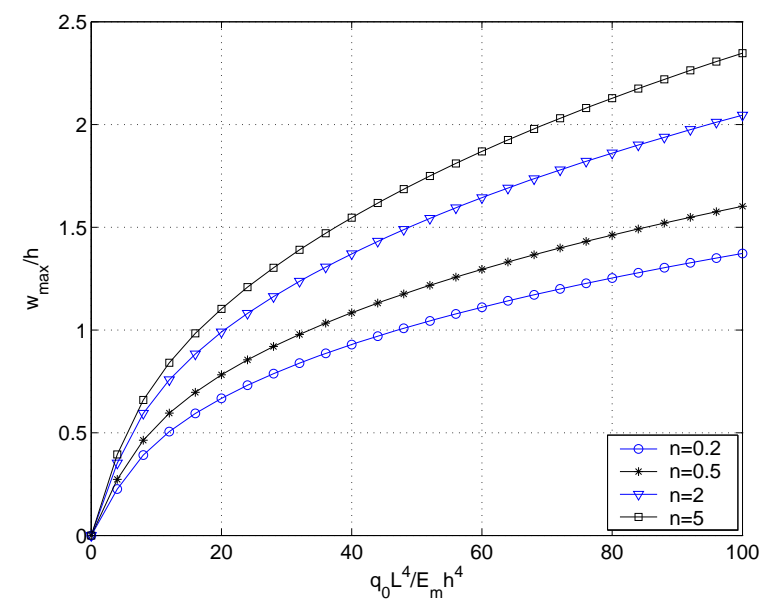

Fig. 8. Relation between normalized load and dimensionless maximum deflection of SS beam

(a)

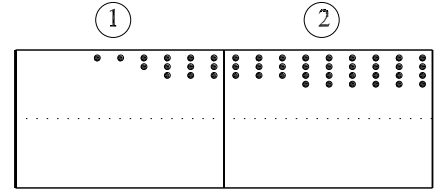

element number

(b)
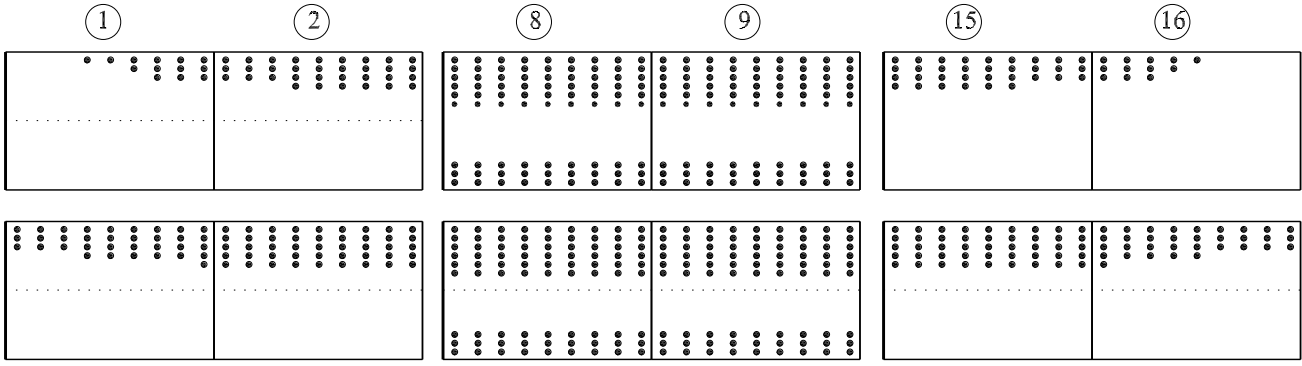

(c)
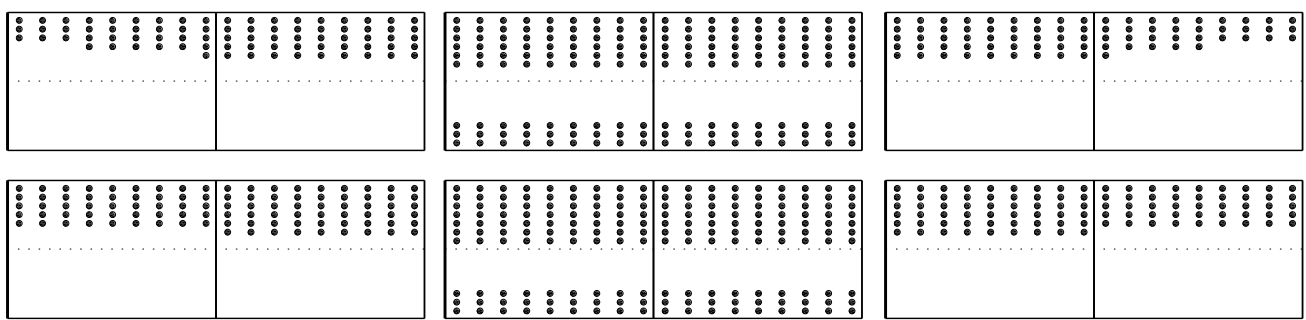

- Gauss points where yielding occurs

Fig. 9. Plastic zone in selected elements of SS beam under uniform loading:

(a) $q_{0}=4000 \mathrm{kN}$, (b) $q_{0}=6000 \mathrm{kN}$; (c) $q_{0}=8000 \mathrm{kN}(n=2)$ 
index $n$. The propagation of plastic zone in the SS beam during the loading process is shown in Fig. 9 for various values of the load amplitude. The size of the plastic zone gradually increases by increasing the load amplitude. Different from the CC beam, the plastic zone in the middle region of the SS beam is much larger than that of the CC beam. A detail examination of the stresses at Gauss points (not shown herein) also confirms that the first yielding in the SS beam occurs in the layer close to the ceramic earlier that it does in the layer next to the metal surface.

\subsection{Cantilever beam under an eccentric axial load}

A cantilever FG beam with $L=5 \mathrm{~m}, b=0.2 \mathrm{~m}, h=0.1 \mathrm{~m}$, subjected to an eccentric compressive axial load $\mathrm{P}$ at its free end is considered. The material data for the constituents are adopted from the work of Jahromi et al. [16] as follows: $E_{c}=80 \mathrm{GPa}$, $E_{m}=56 \mathrm{GPa}, \sigma_{0}=106 \mathrm{MPa}, E_{0}=12 \mathrm{GPa}$ and $q=17.2 \mathrm{GPa}$.

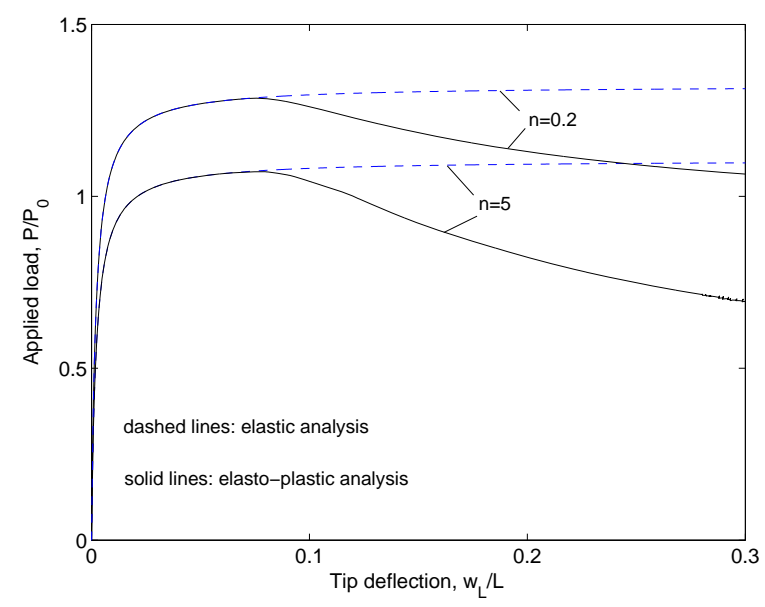

Fig. 10. Equilibrium paths for $\mathrm{CF}$ beam under eccentric axial load $\left(r_{c}=0.01\right)$

In Fig. 10, the equilibrium paths for the CF beam obtained by elastic and elastoplastic analyses are depicted for an eccentric ratio $r_{c}=0.01$ and two values of the powerlaw index, $n=0.2$ and $n=5$. The effect of the eccentric ratio $r_{c}$ on the load-displacement curve of the beam is displayed in Fig. 11 for $n=2$. The eccentric ratio is defined as $r_{c}=\frac{e c}{r}$, where $e$ is distance from the applied load to the neutral surface, $c=h / 2-h_{0}$, and $r=\sqrt{I / A}$ is the radius of gyration of the beam [30]. The applied load is normalized by the Euler load of a pure metal cantilever beam, namely $P_{0}=\pi^{2} E_{m} I / 4 L^{2}$. As seen from Fig. 10, the plastic deformation changes the post-buckling behavior of the beam, and the post-buckling of the beam becomes unstable when the effect of the plastic deformation is taken into account. The post-buckling strength measured in term of the ratio between the applied load and the buckling load is affected by the power-law index $n$, and the post-buckling strength of the beam associated with a higher index $n$ is weaker. The limit load of the beam, as seen from Fig. 11, gradually reduces by raising the eccentric ratio. 


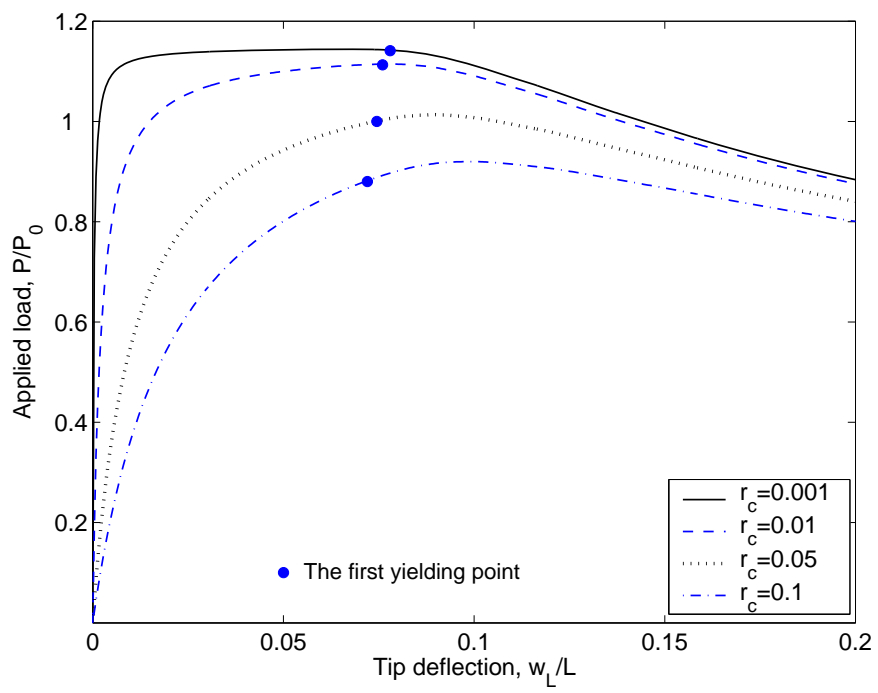

Fig. 11. Effect of eccentric ratio on load-displacement curve of CF beam $(n=2)$

In addition, the deflection of the at which the first yielding occurs is smaller for a large eccentric ratio. The effect of plastic deformation on the behavior of the FG beam in postbuckling region mentioned above is in agreement with the result previously shown in Ref. [22].

\section{CONCLUSIONS}

A finite element procedure for elasto-plastic analysis of FG ceramic-metal beams was presented. A bilinear stress-strain model is assumed for the metal phase and the effective elasto-plastic properties of the FGM were evaluated by using TTO model. Based on Euler-Bernoulli beam theory, a nonlinear beam element, taking the effect of plastic deformation into account, was formulated and employed in the analysis. An incrementaliterative procedure based on Newton-Raphson method was employed in solving the nonlinear equilibrium equations and tracing the equilibrium paths. Numerical examples have been given to show the accuracy and efficiency of the derived formulation. The numerical results have shown that yielding in the FG beam commences at the layer close to the ceramic surface much earlier that it does at the layer near the metal surface. The formation and propagation of the plastic zones in the FG beam, which are quite different from homogeneous beams, have also been examined and highlighted.

\section{ACKNOWLEDGEMENT}

This research is funded by Vietnam National Foundation for Science and Technology Development (NAFOSTED) under Grant Number 107.02-2015.02. The authors gratefully thank the Reviewers for their valuable comments and suggestions to improve the quality of the paper. 
Nguyen Dinh Kien, Tran Thi Thom, Sergey Alexandrov, Le Thi Ha

\section{REFERENCES}

[1] V. Birman and L. W. Byrd. Modeling and analysis of functionally graded materials and structures. Applied Mechanics Reviews, 60, (5), (2007), pp. 195-216. doi:10.1115/1.2777164.

[2] A. Chakraborty, S. Gopalakrishnan, and J. N. Reddy. A new beam finite element for the analysis of functionally graded materials. International Journal of Mechanical Sciences, 45, (3), (2003), pp. 519-539. doi:10.1016/s0020-7403(03)00058-4.

[3] R. Kadoli, K. Akhtar, and N. Ganesan. Static analysis of functionally graded beams using higher order shear deformation theory. Applied Mathematical Modelling, 32, (12), (2008), pp. 2509-2525. doi:10.1016/j.apm.2007.09.015.

[4] Y. A. Kang and X. F. Li. Bending of functionally graded cantilever beam with power-law non-linearity subjected to an end force. International Journal of Non-Linear Mechanics, 44, (6), (2009), pp. 696-703. doi:10.1016/j.ijnonlinmec.2009.02.016.

[5] Y. A. Kang and X. F. Li. Large deflections of a non-linear cantilever functionally graded beam. Journal of Reinforced Plastics and Composites, 29, (12), (2010), pp. 1761-1774. doi:10.1177/0731684409103340.

[6] Y. Huang and X. F. Li. Buckling analysis of nonuniform and axially graded columns with varying flexural rigidity. Journal of Engineering Mechanics, 137, (1), (2010), pp. 73-81. doi:10.1061/(asce)em.1943-7889.0000206.

[7] C. A. Almeida, J. C. R. Albino, I. F. M. Menezes, and G. H. Paulino. Geometric nonlinear analyses of functionally graded beams using a tailored Lagrangian formulation. Mechanics Research Communications, 38, (8), (2011), pp. 553-559. doi:10.1016/j.mechrescom.2011.07.006.

[8] S. Taeprasartsit. A buckling analysis of perfect and imperfect functionally graded columns. Proceedings of the Institution of Mechanical Engineers, Part L: Journal of Materials Design and Applications, (2011), p. 1464420711426686. doi:10.1177/1464420711426686.

[9] S. V. Levyakov. Elastica solution for thermal bending of a functionally graded beam. Acta Mechanica, 224, (8), (2013), p. 1731. doi:10.1007/s00707-013-0834-1.

[10] D. G. Zhang. Nonlinear bending analysis of FGM beams based on physical neutral surface and high order shear deformation theory. Composite Structures, 100, (2013), pp. 121-126. doi:10.1016/j.compstruct.2012.12.024.

[11] D. K. Nguyen. Large displacement response of tapered cantilever beams made of axially functionally graded material. Composites Part B: Engineering, 55, (2013), pp. 298-305. doi:10.1016/j.compositesb.2013.06.024.

[12] D. K. Nguyen. Large displacement behaviour of tapered cantilever Euler-Bernoulli beams made of functionally graded material. Applied Mathematics and Computation, 237, (2014), pp. 340-355. doi:10.1016/j.amc.2014.03.104.

[13] D. K. Nguyen and B. S. Gan. Large deflections of tapered functionally graded beams subjected to end forces. Applied Mathematical Modelling, 38, (11), (2014), pp. 3054-3066. doi:10.1016/j.apm.2013.11.032.

[14] I. Tamura, Y. Tomota, and M. Ozawa. Strength and ductility of Fe-Ni-C alloys composed of austenite and martensite with various strengths. In Proceedings of the Third International Conference on Strength of Metals and Alloys, Vol. 1, Institute of Metals and Iron and Steel Institute, London, (1973). pp. 611-615.

[15] R. Gunes, M. Aydin, M. K. Apalak, and J. N. Reddy. The elasto-plastic impact analysis of functionally graded circular plates under low-velocities. Composite Structures, 93, (2), (2011), pp. 860-869. doi:10.1016/j.compstruct.2010.07.008. 
[16] B. H. Jahromi, H. Nayeb-Hashemi, and A. Vaziri. Elasto-plastic stresses in a functionally graded rotating disk. Journal of Engineering Materials and Technology, 134, (2), (2012), p. 021004. doi:10.1115/1.4006023.

[17] H. Huang and Q. Han. Elastoplastic buckling of axially loaded functionally graded material cylindrical shells. Composite Structures, 117, (2014), pp. 135-142. doi:10.1016/j.compstruct.2014.06.018.

[18] H. Huang, B. Chen, and Q. Han. Investigation on buckling behaviors of elastoplastic functionally graded cylindrical shells subjected to torsional loads. Composite Structures, 118, (2014), pp. 234-240. doi:10.1016/j.compstruct.2014.07.025.

[19] Y. Zhang, H. Huang, and Q. Han. Buckling of elastoplastic functionally graded cylindrical shells under combined compression and pressure. Composites Part B: Engineering, 69, (2015), pp. 120-126. doi:10.1016/j.compositesb.2014.09.024.

[20] G. Nie and Z. Zhong. Exact solutions for elastoplastic stress distribution in functionally graded curved beams subjected to pure bending. Mechanics of Advanced Materials and Structures, 19, (6), (2012), pp. 474-484. doi:10.1080/15376494.2011.556835.

[21] H. Mahbadi. Elastoplastic analysis of functionally graded beams under mechanical loads. Iranian Journal of Mechanical Engineering Transactions of the ISME, 13, (2), (2012), pp. 35-58.

[22] D. K. Nguyen, B. S. Gan, T. H. Trinh, and S. Alexandrov. Post-buckling behavior of elasticplastic functionally graded beams subjected to eccentric axial load. Journal of Multidisciplinary Engineering Science and Technology, 2, (2015), pp. 1129-1135.

[23] Z. H. Jin, G. H. Paulino, and R. H. Dodds. Cohesive fracture modeling of elastic-plastic crack growth in functionally graded materials. Engineering Fracture Mechanics, 70, (14), (2003), pp. 1885-1912. doi:10.1016/s0013-7944(03)00130-9.

[24] C. Chebl and K. W. Neale. A finite element method for elastic-plastic beams and columns at large deflections. Computers $\mathcal{E}$ Structures, 18, (2), (1984), pp. 255-261. doi:10.1016/00457949(84)90123-8.

[25] C. Cichoń. Large displacements in-plane analysis of elastic-plastic frames. Computers \& Structures, 19, (5), (1984), pp. 737-745. doi:10.1016/0045-7949(84)90173-1.

[26] M. A. Crisfiled. Nonlinear finite element analysis of solids and structures. Volume 1: Essentials. John Wiley \& Sons, Chichester UK, (1991).

[27] D. K. Nguyen, T. H. Trinh, and B. S. Gan. Post-buckling response of elastic-plastic beam resting on an elastic foundation to eccentric axial load. The IES Journal Part A: Civil \& Structural Engineering, 5, (1), (2012), pp. 43-49. doi:10.1080/19373260.2012.652769.

[28] R. D. Cook. Concepts and applications of finite element analysis. John Wiley \& Sons, New York, 3rd edition, (1989).

[29] K. J. Bathe and M. Kojic. Inelastic analysis of solids and structures. Springer, (2005).

[30] J. M. Gere and S. Timoshenko. Mechanics of materials. Chapman \& Hall, 3rd SI edition, (1991). 